\title{
Checklisten als Safety-Tool im Schockraum und Operations- saal - ein Gewinn an Sicherheit für Patient und Personal
}

\author{
Christoph G. Wölfl*, Christina Huwer*, Gerrit Matthes*, Axel Ekkernkamp, \\ Paul Alfred Grützner, Matthias Münzberg*
}

\section{Zusammenfassung}

In der Rettungskette der „Schwerverletztenversorgung“ befinden sich die Akteure um den Patienten an einem Hochsicherheitsarbeitsplatz. Bis zu 140 Personen sind an der Versorgung eines schwerverletzten Patienten beteiligt. 140 Personen mit unterschiedlicher technischer Kompetenz, unterschiedlicher Verfahrenskompetenz und v.a. unterschiedlichen „soft skills“ und damit interpersoneller und interprofessioneller Kompetenz.

\section{Einleitung}

Die Folge ist, dass, trotz guter Medizin und guten Ablaufkenntnissen, sowohl in der Präklinik als auch in der Klinik und dort im Schockraum eine für den Patienten ggf. letale oder schädigende Situation entsteht und für die Handelnden eine frustrierende und erschreckende Erfahrung zurückbleibt.

\section{Analyse}

Die BG Klinik Ludwigshafen hat aktuell eine ATLS(Advanced Trauma Life Support-)Schulungsrate in der Unfallchirurgie von $95 \%$, in der Anästhesie von $60 \%$. In der Pflege haben wir zunehmend ATCN-(Advanced Trauma Care for Nurses-)geschulte Mitarbeiter im Schockraum. Dennoch ergeben sich Schockraumabläufe, die nicht nach den festgelegten Kriterien ablaufen.

Warum? Eine Umfrage unter allen Mitarbeiterinnen und Mitarbeitern, die im

OP-JOURNAL 2014; 30: 4-6

(c) Georg Thieme Verlag KG Stuttgart · New York DOI http://dx.doi.org/10.1055/s-0034-1368539

Checklists as Safety Tool in the Shock Room and Operating RoomIncreased Security for Patient and Personnel

In the rescue chain of "management of trauma patients" the actors around the patient are in a high-security working area. Up to 140 people participate in the management of a severely traumatised patient. That is 140 people with differing technical competances and, above all, differing "soft skills", thus possessing interpersonnel and interprofessional competences.
Trifft sich bspw. das Schockraumteam wie vorgesehen nach Alarmierung innerhalb von 10 Minuten, besteht eine durchschnittliche Wartezeit auf den Patienten von 5-10 min. Diese 10 Minuten „überlebt“ die Awareness. Wenn es zu einer Verzögerung kommt, beginnt zunächst eine Konversation im Team ausgehend von der Frage nach dem Eintreffen, hin zu persönlichen, oft auch privaten Gesprächen, und die Awareness für die Situation „Schockraum“ lässt rapide nach. Die Anspannung schlägt in Gelassenheit um. Die Ausgangssituation für das Team und den Patienten wird schlechter.

Wie kann die Kommunikation und Awareness verbessert werden und damit eine Fehlerreduktion in der kritischen Situation Schockraumversorgung erreicht werden?

- „Es ist viel zu laut.“

- „Es sind zu viele Leute im Schockraum."

- „Manchmal wissen wir gar nicht, was genau los ist, und der Patient rauscht an einem vorbei.“

- „Was hat der Patient genau und warum wird wie entschieden?"

- „Als Pflegekraft habe ich den Eindruck, nur die Ärzte wissen letztendlich, wie es weiter geht - das frustriert.“

- „Wer ist wer - plötzlich sind neue Mitarbeiter dabei, die keiner kennt."

Diese Aussagen repräsentieren klassische Verstärker eines möglichen Handlungsabbruchs innerhalb eines Teams:

- laute Umgebung

- viele Personen

- keine Kommunikationsstruktur

Analysiert man solche Situationen in der Luftfahrt, sind dies Ausgangssituationen für viele Unfälle. Um dies zu vermeiden, ist die sogenannte „Awareness“ der maßgebliche Faktor. Im Team muss eine Grundaufmerksamkeit vorhanden sein, welche das Team „am“ Geschehen hält.
Hierzu kann man einiges aus dem OP lernen.

\section{Time-out im Operationsaal}

Im Jahr 2012 wurden in Deutschland 1137 Sterbefälle nach Komplikationen bei chirurgischen Eingriffen und medizinischer Behandlung dokumentiert. Hinzu kommt die kaum messbare Zahl der bei Operationen aufgetretenen Komplikationen, die nicht zum Tod, aber zu einem bleibenden Gesundheitsschaden führen.

Ein Großteil dieser Komplikationen kann mit geringem Aufwand vermieden werden. Dies hatte auch die Weltgesundheitsorganisation (WHO) erkannt und im Jahr 2007 die „Save Surgery Saves Lives"-Kampagne gestartet. Mit diesem Projekt brachte die WHO Experten aller bei Operationen beteiligten Professionen wie Chirurgen, Anästhesisten und $\mathrm{OP}$ Pflegepersonal zusammen, um eine Strategie zur Verbesserung der Sicherheit bei

\footnotetext{
* ATLS Research Group - AUC Deutschland.
} 
Operationen zu finden. Eines der wesentlichen Ergebnisse dieser Initiative war die Entwicklung der Surgical Safety Checklist, die 2009 veröffentlicht wurde.

Die Entwicklung immer weiterer medizinischer Checklisten gründet sich auf den Erfolg der Surgical Safety Checklist. In einer Pilotuntersuchung in Krankenhäusern rund um die Welt konnte gezeigt werden, dass lediglich durch die Implementation dieser Checkliste die Rate perioperativer Komplikationen sowohl in den Industrienationen als auch in Schwellen- und Entwicklungsländern signifikant gesenkt werden konnte. In den Schwellen- und Entwicklungsländern konnte darüber hinaus sogar eine Senkung der Mortalitätsrate erreicht werden [5].

Das Prinzip der Checkliste beruht auf der Idee, einen Vorgang wie eine Operation in kleinere Komponenten zu unterteilen und so Fehler durch Vergessen oder Unterlassen zu vermeiden.

Die Nutzung der Surgical Safety Checkliste erfordert jeweils etwa eine Minute vor der Einleitung der Anästhesie, vor dem Beginn der eigentlichen Operation und bevor der Patient den OP-Saal verlässt (http://whqlibdoc.who.int/publications/2009/9789241598590_eng_Checklist.pdf?ua=1). Vor Beginn der Einleitung überprüft der Anästhesist anhand der Checkliste u. a., dass es sich um den richtigen Patienten handelt, dass die zu operierende Seite korrekt markiert wurde, ob Allergien bekannt sind und dass das Narkosegerät ordnungsgemäß getestet wurde. Außerdem werden Probleme, wie ein schwieriger Atemweg oder eine zu erwartende schwere Blutung, antizipiert. Der Chirurg stellt vor Beginn der Operation sicher, dass alle Mitglieder des Teams einander kennen, dass es sich um den richtigen Patienten und die korrekte Seite handelt und dass die perioperative Antibiotikaprophylaxe verabreicht wurde. Außerdem wird gefordert, dass sich das gesamte Team, also Chirurgen, Anästhesisten und OP-Pflege, im Vorfeld über kritische Schritte der Operation und eventuell zu erwartende Komplikationen austauscht. Bevor der Patient den OP-Saal verlässt, bestätigt das OP-Pflegepersonal u.a., dass die Zählkontrolle stimmt und dass eventuell entnommene Proben korrekt beschriftet wurden.

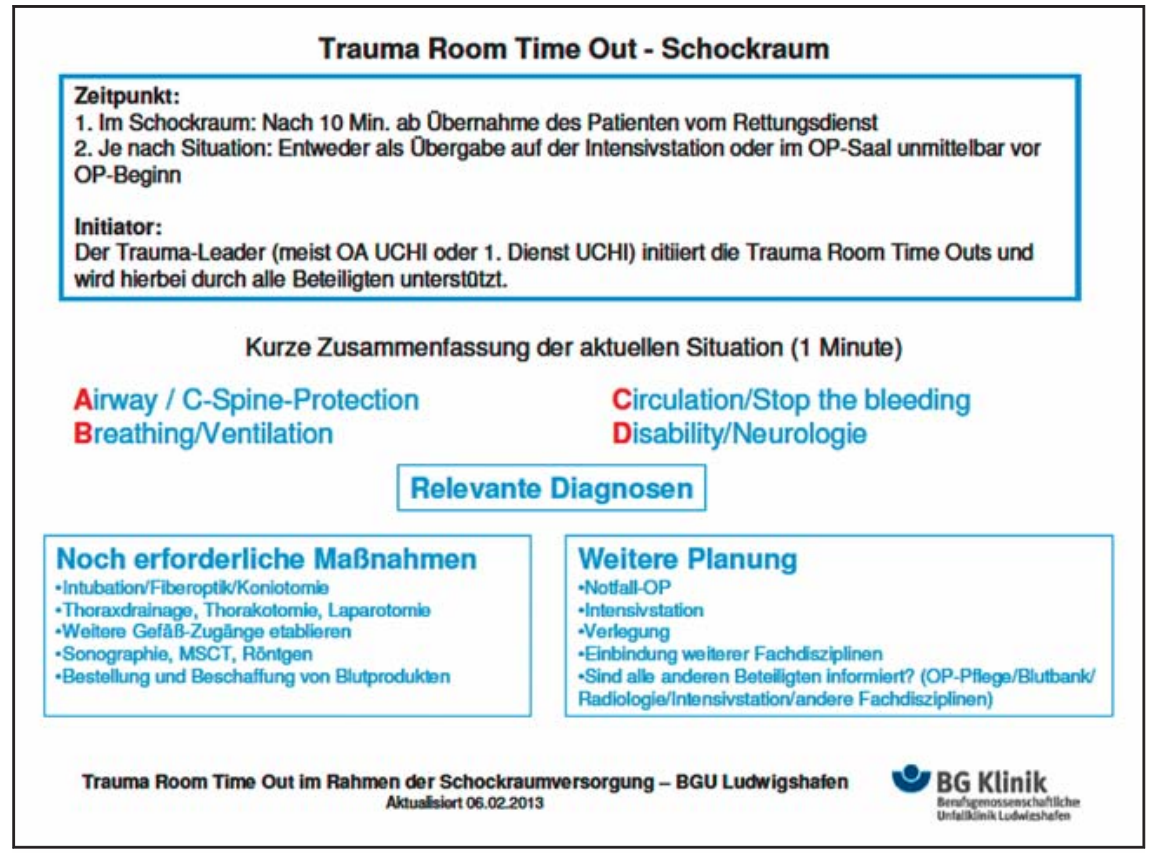

Abb. 1 Trauma Room Time Out (TRTO ${ }^{\circledR}$ BG Unfallklinik Ludwigshafen) - Ablauf.

Aber die elektive Operation ist ein vergleichsweise einfaches Szenario. Die Kontrollinstanzen beginnen bereits mit der Aufnahme des Patienten auf die Station, und nach Abarbeiten der Checklisten dort, im Rahmen der Narkoseeinleitung und vor OP-Beginn entsteht eine hohe Sicherheit.

Aber auch hier ist die Frage nach dem aktiven Leben wichtig. Weiß jeder im Team wirklich immer, wer gerade Narkose macht und wie der Saalspringer heißt auch wenn das Team meist den Tag zusammen im Saal verbringt?

Im „kritischen“ Szenario der Schockraumversorgung herrschen Zeitdruck und ein hoher Workload - es trifft immer ein neues Team zusammen mit unterschiedlichen Fertigkeiten und Fähigkeiten sowie Soft Skills. Viel mehr als im normalen Operationsablauf sind hier Fehler und Kommunikationsdefizite vorprogrammiert - welche es zu verhindern gilt.

In Ludwigshafen wurde als Baseline mit allen beteiligten Disziplinen ein 2-tägiges Coaching-Seminar durchgeführt. Trotz hervorragender Infrastruktur und hohem Ausbildungsstand wurden Schwachpunkte identifiziert. Nah- und Fernziele für die Optimierung der interdisziplinären Schockraumversorgung wurden definiert. Die wichtigsten Punkte waren die Optimierung der Kommunikation und der gemeinsamen Entscheidungsfindung.
Das „Team Time-out“ im Operationssaal ermöglicht zu Beginn der Operation einen nochmaligen Abgleich des gemeinsamen Wissensstands über den Patienten und evtl. zu erwartender Probleme. Die Etablierung einer ebensolchen Sicherheitskultur im Schockraum wurde als Ziel definiert.

\section{TRTO}

In Anlehnung an das Team Time-out wurde der sogenannte „Trauma Room Time Out“" TRTO entwickelt (Abb. 1). Im Folgenden wird der Ludwigshafener Schockraumablauf mit den integrierten Time-outs - die an jedem wichtigen Entscheidungszeitpunkt eingebaut wurden - vorgestellt.

1. Alarmierung "Schockraumalarm“ durch den 1. Chirurgischen Dienst (Traumahandy). Ohne Rückfragen begeben sich die jeweiligen Mitarbeiter innerhalb von $10 \mathrm{~min}$ in den Schockraum.

2. Der 1. Chirurgische Dienst leitet die Vorstellung ein und führt ein Briefing des Teams über den angemeldeten Schockraum durch, die Leitung wird an den Traumaleader (OA Unfallchirurgie) übergeben.

3. Mit dem Eintreffen des Patienten wird eine digitale Uhr aktiviert. Der 1. Chirurgische Dienst stellt sich vor und bittet um Übergabe des Patienten durch den Notarzt. Der Patient verbleibt auf der Rettungsdiensttrage. Ein vorheriges Umlagern würde die Aufmerksam- 
keit einzelner Teammitglieder, die dann z.B. die Beatmung wechseln etc., ablenken.

4. Nach der Übergabe nach ABCDE-Kriterien wiederholt der 1 . Chirurgische Dienst die Übergabe laut (Readback). Der Traumaleader notiert die Ausgangswerte auf einem Whiteboard. Somit haben alle einen ersten gemeinsamen Wissensstand. Der Primary Survey (Erstuntersuchung) beginnt. Der Rettungsdienst verlässt den Schockraum.

5. Sobald der Primary Survey beendet und der Patient initial stabilisiert ist, erfolgt durch den Traumaleader ein 1. TRTO. Nach ABCDE-Kriterien wird der aktuelle Stand laut abgefragt und festgelegt ob akut im Schockraum noch Maßnahmen nötig sind oder der Patient in das MSCT, den OP oder auf die Intensivstation gebracht werden kann bzw. muss. Sollte der Primary Survey länger als $12 \mathrm{~min}$ dauern, wird der Traumaleader durch eine Signalton der Digitaluhr $12 \mathrm{~min}$ nach Beginn der Versorgung (2 min Übergabe, 10 min Primary Survey) auf die Durchführung des TRTO aufmerksam gemacht.

6. Der 2. TRTO erfolgt nach Durchführung des MSCT,
7. der 3. bei Übergabe an das Notfalloperationsteam oder auf der Intensivstation.

\section{Zusammenfassung und Ausblick}

Neben der klinischen Einführung ist die wissenschaftliche Aufarbeitung des TRTO von großem Interesse. Die ATLSResearch-Gruppe des Deutschen ATLS Programms plant aktuell einer Multicenterstudie zum Nutzen des TRTO. Weiterhin besteht bereits eine Zusammenarbeit mit der WHO auf diesem Gebiet.

Der TRTO sollte ein fester Bestandteil im Rahmen der Schockraumversorgung sein. Die Implementierung in bestehende bzw. zu entwickelnde Simulationskurse erscheint sinnvoll. Mehr Wissen über den Patienten bedeutet mehr Sicherheit für Patient und Handelnde. Somit ist der TRTO ein wichtiges Sicherheitstool für die kritische Situation am Hochsicherheitsarbeitsplatz Schockraum.

Die Publikation enthält Auszüge aus Wölfl C, Kotter J, Trupkovic T, Grützner PA, Münzberg M. Trauma Room Time Out (TRTO) Neues Sicherheitstool zur Verbesserung der Patientensicherheit und Mitarbeiterzufriedenheit im Schockraum. Unfallchirurg 2014; 117(1): 83-85 (mit freundlicher Genehmigung von Springer Science + Business Media).

\section{Literatur}

${ }^{1}$ Rydenfällt C, Ek A, Larsson PA. Safety checklist compliance and false sense of safety: new directions for research. BMJ Qual Saf 2014; 23 : 183-186

2 Böhmer AB, Wappler F, Tinschmann T et al. The implementation of a perioperative checklist increases patients' perioperative safety and staff satisfaction. Acta Anaesthesiol Scand 2012; 56: 332-338

${ }^{3}$ Treadwell JR, Lucas S, Tsou AY. Surgical checklists: a systematic review of impacts and implementation. BMJ Qual Saf 2014; 23: 299318

${ }^{4}$ Nance JJ. Why Hospitals Should Fly: The Ultimative Flight Plan to Patient Safety and Quality Care. Bozeman, MT: Second River Healthcare Press; 2008

${ }^{5}$ Statistisches Bundesamt. Todesursachenstatistik. Wiesbaden; 2013

${ }^{6}$ Haynes AB, Weiser TG, Berry WR et al. A surgical safety checklist to reduce morbidity and mortality in a global population. N Engl J Med 2009; 360: 491-499

7 Wölfl C, Kotter J, Trupkovic $T$ et al. Trauma Room Time Out (TRTO) Neues Sicherheitstool zur Verbesserung der Patientensicherheit und Mitarbeiterzufriedenheit im Schockraum. Unfallchirurg 2014; 117: 83-85

\section{Dr. med. Christoph Wölfl}

Geschäftsführender Oberarzt

Dr. med. Matthias Münzberg

Assistenzarzt

Prof. Dr. med. P. A. Grützner

Ärztlicher Direktor

Klinik für Unfallchirurgie und

Orthopädie

BG Klinik Ludwigshafen

Ludwig-Guttmann-Str. 13

67071 Ludwigshafen

\section{Dr. med. Christina Huwer \\ Assistenzärztin}

Prof. Dr. med. Gerrit Matthes

Oberarzt

Prof. Dr. med. Axel Ekkernkamp

Ärztlicher Direktor

Klinik für Unfallchirurgie und

Orthopädie

Unfallkrankenhaus Berlin

Warener Str. 7

12683 Berlin

christoph.woelfl@bgu-ludwigshafen.de 